Jasmin Schreyer, Jan-Felix Schrape

\title{
Plattformzentrierte Arbeitskoordination im kommerziellen und kooperativen Fahrradkurierwesen
}

https://doi.org/10.1515/arbeit-2021-0020

Zusammenfassung: Dieser Aufsatz vergleicht den Einsatz digitaler Plattformen in kommerziellen und kooperativen Arbeitszusammenhängen. Auf der Basis zweier qualitativer Fallstudien zu dem automatisierten Arbeitsmanagement in der kommerziellen Gig Economy (Lieferando.de) und der kollektiven Nutzung informationstechnischer Plattformen in kooperativen Projekten (Crow Cycle Courier Collective) diskutieren wir das veränderte Zusammenspiel von technischen und sozialen Strukturierungsleistungen in der Koordination von Arbeit.

Schlüsselwörter: Plattformarbeit, Gig Economy, algorithmisches Management, kooperatives Management

\section{Platform-centric work coordination in the commercial and cooperative bicycle courier sector}

\begin{abstract}
The article compares the use of digital platforms in the gig economy and in cooperative work contexts. On the basis of two qualitative case studies on commercial and cooperative forms of platform labor in the bicycle courier sector, we discuss the changing interplay of technical and social structuring services in the coordination of work.
\end{abstract}

Keywords: Platform labor, gig economy, algorithmic management, collective management

Jasmin Schreyer, Friedrich-Alexander-Universität Erlangen-Nürnberg, Institut für Soziologie, Nuremberg Campus of Technology, Fürther Str. 246 c, 90429 Nürnberg, Deutschland. PD Dr. Felix Schrape, Universität Stuttgart, Institut für Sozialwissenschaften, Seidenstr. 35, 70174 Stuttgart, Deutschland.E-Mails: jasmin.schreyer@fau.de, felix.schrape@sowi.uni-stuttgart.de 


\section{Einleitung}

Die Digitalisierung hat eine substanzielle Rekonfiguration der gesellschaftlichen Koordinationsweisen angestoßen. Seit einigen Jahren tritt dabei auch auf dem Feld der Erwerbsarbeit die ,Plattform“ als „, digital service that facilitates interactions between two or more distinct but interdependent sets of users" (OECD 2019, 21) in den Vordergrund der Wahrnehmung. In diesem Aufsatz nehmen wir den Einsatz von digitalen Plattformen in kommerziellen und kooperativen Arbeitszusammenhängen in den Blick. Ausgehend von zwei problemzentrierten Fallstudien zu dem algorithmischen Arbeitsmanagement in der kommerziellen Gig Economy am Beispiel von Lieferando.de sowie der kollektiven Nutzung informationstechnischer Plattformen in kooperativen Arbeitszusammenhängen am Beispiel des Crow Cycle Courier Collective diskutieren wir das veränderte Zusammenspiel von technischen und sozialen Strukturierungsleistungen in der Koordination von Arbeit. In einer anschließenden vergleichenden Gegenüberstellung arbeiten wir die Gemeinsamkeiten und Unterschiede beider Fallbeispiele heraus und diskutieren die Spezifika der plattformvermittelten Arbeitskoordination in kommerziellen und kooperativen Arbeitszusammenhängen. Wir beginnen im Folgenden mit einer einführenden Übersicht zu den sozialwissenschaftlichen Debatten um die Plattformisierung von Arbeit.

\section{Plattformisierung der Arbeitskoordination}

Aus der bisherigen Koevolution von Technik und Gesellschaft lassen sich drei basale Eigenheiten soziotechnischer Infrastrukturen ableiten, die sich auch auf digitale Plattformen und deren Effekte auf dem Feld der Arbeit übertragen lassen (Dolata/Schrape 2018; Schrape 2021):

- Erstens haben technische Infrastrukturen ermöglichende Eigenheiten: Sie vergrößern gesellschaftliche Gestaltungsmöglichkeiten und individuelle Erfahrungsräume, effektivieren Produktionszusammenhänge und vereinfachen kollektive Abstimmungsprozesse.

- Zweitens bilden technische Infrastrukturen strukturierende und kanalisierende Eigenheiten aus: Durch ihre Koordinationsleistungen strukturieren sie individuelle, kollektive und organisationale Beziehungsmuster und prägen Arbeits- und Austauschzusammenhänge.

- Drittens erweitern technische Infrastrukturen die Möglichkeiten sozialer Kontrolle: Durch ihre Strukturierungs- und Kanalisierungsleistungen er- 
leichtern sie die systematische Beobachtung und die positive oder negative Sanktionierung von Erwerbstätigen.

Alle diese Eigenheiten sind allerdings nicht durch Technik per se festgelegt. Sie sind das Resultat vielfältiger sozialer Aneignungsprozesse, die in langfristige gesellschaftliche Entwicklungslinien eingelassen sind. Insbesondere die Erwerbsarbeit unterliegt schon seit der beginnenden Industrialisierung einer verstärkten Technisierung, die zu der Entstehung neuer soziopolitischer Konfliktlinien beigetragen hat (Pfeiffer 2018). Die dadurch angestoßenen Aushandlungsprozesse mündeten in der BRD ab den 1950er Jahren in die Etablierung des ,Normalarbeitsverhältnisses', das sich durch unbefristete Vollzeitbeschäftigung und soziale Absicherung auszeichnet. Schon ab den 1980er Jahren wurden angesichts weiterer technischer Neuerungen allerdings erste Diagnosen zu einer Erosion dieses Normalarbeitsverhältnisses formuliert; in den nachfolgenden Debatten wurden bereits viele Dynamiken thematisiert, die im Digitalisierungsdiskurs erneut hervortreten, darunter eine Prekarisierung von Arbeit sowie eine Entgrenzung zwischen Erwerbsarbeit und Privatleben (Trinczek 2011).

Insbesondere die noch jungen Phänomene plattformkoordinierter Arbeit wurden zuletzt umfassend diskutiert (De Stefano 2016; Kuhn/Galloway 2019; Healy u.a. 2020) und - obgleich sie in der BRD noch eine eher geringe Relevanz für den Arbeitsmarkt haben (Schäfer 2020) - mitunter als ein Prototyp für künftige Arbeitsformen im Allgemeinen angesehen (Lücking 2019). Autoren wie Jeremy Rifkin $(2014,17)$ schrieben der plattformkoordinierten Arbeit dabei unter dem Stichwort ,Sharing Economy' zunächst das Potenzial zu, eingespielte Ressourcenverteilungen aufzubrechen und eine „dritte industrielle Revolution“ auszulösen, die in eine Demokratisierung ökonomischer Verhältnisse münden sollte. Auch weniger radikal orientierten Studien galt die "Sharing Economy" „als Sinnbild für die neuen, internetbasierten Geschäftsmodelle“, in denen plattformbetreibende Unternehmen nicht mehr über eigene Produktionsmittel verfügen, sondern diese von den Erwerbstätigen eingebracht werden (Eichhorst/Spermann 2015, 4). Damit waren von Beginn an „einerseits Hoffnungen auf mehr Wachstum und mehr Beschäftigung und andererseits Sorgen vor unsicheren Beschäftigungsverhältnissen“ verbunden (ebd.).

Studien zu bislang erfolgreichen Angeboten in der Plattformökonomie führen indes die ambivalenten Effekte vor Augen, die mit dieser Plattformisierung von ortsunabhängigen und ortsgebundenen Arbeitsmärkten einhergehen (Huws 2014, 2016; Heiland/Brinkmann 2020): Plattformbetreibende Unternehmen akquirieren Dienstleister^innen mit der Aussicht auf eine flexible bzw. eigenverantwortliche Arbeit und definieren sich oft weniger als klassische Ar- 
beitgeber*innen, sondern stellen ihre Position als Intermediäre heraus (van Doorn 2017, 2020; Huws u.a. 2017). Die Arbeitnehmenden verfügen nur über wenige Mitbestimmungsoptionen und werden mit ausdefinierten Plattformregularien konfrontiert. Dieses Zusammenspiel von Flexibilisierung, Standardisierung und Kontrolle in plattformzentrierten Arbeitskontexten lässt sich wie folgt darstellen (Schreyer/Schrape 2018, 2021):

- Flexibilisierung: Neben Möglichkeiten zur flexiblen Einteilung der Arbeitszeiten ist der Zugang zu den plattformvermittelten Arbeitsmärkten vorderhand niederschwellig angelegt. Dienstleisterinnen müssen neben ihrer allgemeinen Geschäftsfähigkeit oft keine weiteren Fähigkeiten angeben. Die Arbeitsmittel müssen allerdings oft selbst eingebracht werden.

- Standardisierung: Dreh- und Angelpunkt für alle Arbeitsabläufe ist die jeweilige Plattform bzw. Smartphone-Applikation. Die Arbeitsvorgänge werden in standardisierte Module zerlegt. Die Möglichkeit, in die Abläufe einzugreifen, ist in der Regel nicht gegeben.

- Kontrolle: Arbeitszeiten, Interaktionen auf der Plattform sowie individuelle Leistungsdaten werden in unternehmenseigenen Datenbanken gespeichert und ausgewertet. Die auf der Plattform aggregierten Daten eröffnen ab einem bestimmten Schwellenwert oft höhere Entlohnungschancen; ein individueller Verhandlungsspielraum ist nicht vorhanden.

Eine solche Integration technisch vermittelter Leistungen (u.a. eine vollautomatisierte Koordination und prozessumspannende Arbeitskontrolle) ist auf dem Feld der Arbeit erst seit der Etablierung mobiler IT-Endgeräte umsetzbar und ermöglicht so zuvor nur in Ausnahmefällen denkbare volatile Arbeitsverhältnisse (Rosenblat/Stark 2016). Vor allen Dingen für Arbeitsmärkte, die vorrangig durch geringfügig Beschäftigte bespielt werden, lässt sich der strukturierende Einfluss proprietärer Plattformen kaum überschätzen. Insbesondere mit Blick auf die plattformzentrierte Koordination von volatiler Arbeit hat sich insofern ein reger Diskurs um Möglichkeiten zur ordnungspolitischen Einhegung entwickelt, die einer Erosion „des historisch gewachsenen Systems der Regulation von Arbeit“ (Boes u.a. 2014, 71) entgegenwirken sollen (Hensel u.a. 2019; Thelen 2018). Aber auch in klassischen betrieblichen Zusammenhängen zeigt sich das ambivalente Wechselspiel von ermöglichenden, kanalisierenden und kontrollierenden Effekten der Plattformisierung (Meyer u.a. 2020; Boes u.a. 2018).

Nichtsdestoweniger bieten neue soziotechnische Konstellationen immer wieder Anlass für vielgestaltige Hoffnungen auf eine Reform sozioökonomischer Verhältnisse. Nachfolgend kontrastieren wir vor diesem Hintergrund das algorithmische Management in der Gig Economy (am Beispiel von Foodora bzw. 
Lieferando.de) mit der Plattformnutzung in kooperativen Projekten (am Beispiel des Crow Cycle Courier Collective), um danach das divergierende Verhältnis von technischen und sozialen Strukturierungsleistungen in diesen Arbeitszusammenhängen aus der Perspektive von Arbeitnehmer^innen herauszuarbeiten.

\section{Algorithmisches Arbeitsmanagement in der Gig Economy}

Der Grundgedanke vieler kommerzieller Plattformangebote fußt - ähnlich den originären Ideen der ,Sharing Economy - auf der Einsicht, dass ein Gutteil der Ressourcen in der modernen Gesellschaft nicht effizient ausgelastet wird. Digitale Plattformen fungieren als Intermediäre auf mehrseitigen Märkten und vermitteln in automatisierter Form zwischen Angebot und Nachfrage (Srnicek 2017; Lehdonvirta 2018; Vallas/Schor 2020). In der wirtschaftsliberalen Idealvorstellung soll so eine Informationssymmetrie zwischen allen Markteilnehmenden erreicht werden (Rosenblat 2018; Ravenelle 2019). Die plattformbetreibenden Unternehmen bauen jedoch zugleich mit jeder Transaktion ihren Pool an Nutzungsdaten aus, der kontinuierlicher Auswertung unterliegt. In der Koordination von Arbeit treten Plattformunternehmen auf diese Weise als so zuvor nicht vorhandene Einflussgrößen hervor (Richardson 2020).

Die Gemeinsamkeit der heterogenen ortsunabhängigen (Cloudwork) und ortsgebundenen (Gigwork) Arbeitszusammenhänge, die sich mit der Plattformökonomie herausgebildet haben, besteht in der zentralen Stellung algorithmischer Strukturen in dem datenbasierten Management bzw. der Koordination von Arbeit sowie der jeweiligen plattformbetreibenden Unternehmen (Rosenblat/Stark 2016; van Doorn 2017, 2020; Kellogg u.a. 2020). Der Arbeitsprozess kann auf diese Weise kleinteilig überwacht und kontrolliert werden, wodurch der Einsatz ungelernter Arbeitskräfte befördert wird. Wie Stefan Kirchner und Elke Schüßler (2019) herausgearbeitet haben, kann das in eine engmaschige ,algorithmische Bürokratie' münden, die zu einer Intensivierung gegebener Rationalisierungstendenzen beiträgt (Zuboff 2018; Kellogg u.a. 2020). Die algorithmische Arbeitskoordination formt durch technikvermittelte Regelsetzungen die Handlungsspielräume der Arbeitskräfte (Duggan u.a. 2020; Schreyer 2021a). Die damit einhergehenden rigiden Kontrollregime erzeugen mitunter allerdings auch selbstorganisierten Widerstand seitens der Arbeitnehmenden (Schreyer 2021b). 
Die Eigenheiten algorithmisch automatisierter Arbeitsorganisation zeigen sich insbesondere hinsichtlich plattformvermittelter Ausprägungen von Gigwork, die mit Blick auf den Zeitaufwand und Anspruch der Tätigkeit über einfache Microjobbing-Tasks hinausgehen. In diesem Bereich haben wir uns exemplarisch mit dem Lieferdienst Foodora beschäftigt, der in der BRD ab 2019 in das Angebot von Lieferando.de übergegangen ist. Diese qualitative Fallstudie ist Teil einer umfassenderen gegenstandsbezogenen Rekonstruktion (dazu: Mayntz 2002, 2009) und basiert neben vielfältigen Dokumentenanalysen (z.B. Websites, Zeitungsberichte, Geschäftsberichte) und der Auswertung eines Chatforums (2014-2020) auf zehn problemzentrierten Interviews mit 16 Fahrer^innen, die für Foodora bzw. Lieferando.de gearbeitet haben. ${ }^{1}$ Die Interviews wurden zwischen November 2017 und Oktober 2020 in drei Städten in Deutschland geführt und dauerten 30 bis 200 Minuten. Drei der 16 Interviewten wurden zweimal (2017/2018 und 2020) interviewt, da sie seit mehreren Jahren für Foodora bzw. Lieferando tätig waren. Acht der Interviewten waren einfache ,Rider'; drei davon nahmen teamleitende Funktionen ein (,Rider Captains'); fünf unserer Gesprächspartner`innen hatten den höchsten Status eines ,Senior Rider Captains“ erreicht. 14 von 16 Interviewten ordneten sich selbst dem männlichen Geschlecht zu; 8 Interviewte wiesen einen Migrationshintergrund auf; das Durchschnittsalter lag bei 23,4 Jahren. Zumindest in der Grundtendenz entsprechen diese Verteilungen der Zusammensetzung der Arbeiterschaft von Foodora bzw. Lieferando.de zum Befragungszeitpunkt.

\subsection{Foodora und Lieferando.de als Arbeitgeber*in}

Foodora wurde 2014 (zunächst unter dem Namen Volo) als Fahrrad-Lieferservice für Restaurants gegründet. Im Frühjahr 2015 wurde das Start-up an das Beteiligungsunternehmen Rocket Internet verkauft; im Herbst 2015 wurde

\footnotetext{
1 Konzeptuelle und empirische Arbeiten werden in problemorientierten Rekonstruktionen im Sinne der ,Grounded Theory“ (Glaser/Strauss 1998; Lamnek 2010, 90 ff.) iterativ ineinander verschränkt. Die eingangs aufgestellten Arbeitshypothesen werden auf der Basis empirischer Beobachtungen fortlaufend spezifiziert und verdichtet, um auf diese Weise verallgemeinerbare Muster zu identifizieren. Auf der Grundlage erster Dokumentenanalysen und Interviews wurde dementsprechend nach dem Prinzip des offenen Kodierens (Strauss/Corbin 1996) ein vorläufiges Kategoriensystem erarbeitet. Im weiteren Verlauf wurden alle Gespräche in Interviewprotokolle mit langen narrativen Passagen überführt. Die Interviewprotokolle wurden danach konsolidiert und in ein umfassendes Fallporträt übertragen. Im Anschluss haben wir die Fallportraits mit der übergreifenden Literaturdiskussion in Bezug gesetzt.
} 
Foodora für 13,2 Mio. Euro an Delivery Hero weiterverkauft. Ende 2018 akquirierte die Unternehmensgruppe Takeaway.com das Deutschlandgeschäft von Delivery Hero und damit auch Foodora, das danach in den bereits operierenden Dienst Lieferando.de überführt wurde. Nachdem Takeaway.com den Mitbewerber Just Eat übernommen hatte, benannte sich das Unternehmen 2020 in Just Eat Takeaway um. Das deutsche Unternehmen Lieferando.de wurde bereits 2014 von Takeaway.com aufgekauft.

Die bis Anfang 2019 eigenständig operierende Plattform Foodora Deutschland unterschied sich in einem wesentlichen Merkmal von anderen Essensauslieferdiensten: Anders als etwa der britische Anbieter Deliveroo und auch anders als Foodora selbst in allen anderen Ländern (Leonardi u.a. 2019; Tassinari/ Maccarrone 2020) beschäftigte Foodora Deutschland seine Fahrer`innen (,Rider`) über feste Arbeitszeitmodelle für geringfügig Beschäftigte. Insgesamt beschäftigte Foodora 2018 zwischen 3.000 und 4.000 Fahrer ${ }^{\star}$ innen sowie 900 Mitarbeiter`innen (Foodora 2018). Dieses Spezifikum rückte Foodora in eine Zwischenposition zwischen Arbeitsplattform und klassischem Unternehmen. Das Unternehmen rechnete sich in seiner Selbstbeschreibung daher auch nicht der Gig Economy zu (Knieps 2021); nichtsdestotrotz wurden arbeitsrechtliche Spielräume kontinuierlich zum Vorteil der Plattform ausgelotet. Just Eat Takeaway führt diese Form der Beschäftigung für Lieferando.de fort und bietet eine stundenbasierte Bezahlung und basale vertragliche Absicherung. 2019 arbeiteten ca. 4.500 Menschen für Lieferando.de, davon 800 im ,Headquarter‘. Im Rahmen der COVID-19-Pandemie hat sich das Angebot von Lieferando.de auf über 20.000 Restaurants ausgedehnt; die Lieferflotte wuchs auf 10.000 Fahrerinnen an. Jedoch ist das Unternehmen weiterhin nicht profitabel (SZ 2021).

Während sich Lieferando.de mit Blick auf die Beschäftigungsverhältnisse von anderen Anbietern abhebt, weist die Plattform in anderen Bereichen Gemeinsamkeiten mit ihnen auf: Zum einen müssen die Fahrerinnen viele Arbeitsmittel (u.a. Smartphones mit Internetverbindung) selbst einbringen; zum anderen sieht Lieferando.de den plattformeigenen Logistik-Algorithmus als Herzstück des Unternehmens an. Niklas Österberg, CEO von Delivery Hero, umschrieb die Zentralstellung informationstechnischer Strukturen in der Gig Economy 2016 wie folgt:

Innovativ ist, wie wir mit unserer Technologie weltweit eine große Anzahl von Restaurants in die Lage versetzen, schneller bessere Gerichte auszuliefern. [...] So versorgen wir die Gastronomen mit Technologie für Bestellaufnahme, Auslieferung oder Tracking. Gleichzeitig helfen wir auch den Kunden bei der Auswahl des richtigen Restaurants auf Grundlage ihrer Essenspräferenzen und zu erwartenden Lieferzeiten. 
Auffällig ist hierbei, dass in der Außenkommunikation von Lieferando.de im Erhebungszeitraum explizit zwischen Kurierfahrer^innen und Mitarbeiter^innen im ,Headquarter' differenziert wurde. Dies kam auch in unseren Interviews zur Sprache: „Wir haben hier so eine Parallelstruktur, die Fahrer sind eigentlich außerhalb des Unternehmens“ (Fahrer^in E 2020). Zwar werden die Fahrer^innen auf den Websites von Just Eat Takeaway als Leistungsträger`innen benannt; die interviewten Fahrer^innen verstanden sich gleichwohl nicht als Teil des eigentlichen Teams (Fahrer*in B 2018). Die Arbeitsbedingungen der Beschäftigten, die an bzw. über dem Algorithmus arbeiten, unterscheiden sich mithin wesentlich von den Arbeitsbedingungen der Beschäftigten, die unter dem Algorithmus arbeiten.

\subsection{Algorithmisches Management als zentrales Koordinationsprinzip}

Die Algorithmen der Plattform garantieren eine effiziente Gestaltung des Arbeitsablaufs. Das zentralisierte algorithmische Management übernimmt die gesamte Koordination und schaltet die Arbeitsschritte sukzessive frei (vgl. für eine eingehendere Betrachtung: Schreyer/Schrape 2021, 2018). Diese Modularisierung erlaubt allen Nutzer^innen der Plattform (Restaurants, Kund`innen, Arbeitnehmende) eine äußerst niederschwellige Partizipation. Sie geht allerdings mit einer hochgradigen Standardisierung einher, die keine Abweichung von den vorgegebenen Pfaden erlaubt. Die Fahrer*innen bleiben so weitgehend ohne Möglichkeit, in den Prozess einzugreifen: „Man bekommt immer nur das Nötigste an Informationen und arbeitet Schritt für Schritt“ (Fahrer*in D 2018). Das bedeutet auch, dass Fahrerinnen kaum zur Lösung etwaiger Probleme im Lieferablauf beitragen können, da sie beispielsweise ihr qualitatives Wissen über regionale Umwelterfordernisse nicht einbringen können.

Dies liegt darin begründet, dass die algorithmischen Strukturen der Plattform vorrangig auf eine vollautomatisierte und prozessumspannende Arbeitsund Ablaufkontrolle entlang standardisierter Kennziffern ausgerichtet sind und das System darüber hinaus keine Kommunikations- und Feedbackschnittstellen zur Verfügung stellt (Ivanova u.a. 2018): Alle Aktivitäten der Fahrer^innen, ihre Verweildauer auf der Plattform bzw. in der Smartphone-Applikation, ihre Durchschnittsgeschwindigkeiten, ihre Reaktionszeiten und weitere Faktoren werden durch die technische Infrastruktur der Plattform registriert, gespeichert und aggregiert. Gleichzeitig bleibt es aus der Sicht der Arbeitnehmer*innen intransparent, nach welchen Kriterien diese Daten weiterverarbeitet werden. Die auf diese Weise objektivierten Datenwerte, die aus dem detaillierten Echtzeit- 
Tracking-System gelesen werden, werden anschließend in visualisierter Form an die Fahrer`innen zurückgespielt. Diese Anwendung spieltypischer Elemente (Gamification) dient vor allem der Motivationssteigerung, berücksichtigt aber weder regionale Unterschiede noch divergente Bedingungen im Straßenverkehr:

Das Ganze basiert auf so 'ner Art Wettbewerbsgedanken. Das ist völliger Unfug, weil wir befinden uns ja im Straßenverkehr. Man kann die Ampel eben nicht einfach mal auf grün stellen, wie man es braucht. Man muss die ganze Zeit achtsam sein, man muss schauen, wie das Wetter ist und wo die Leute wohnen, wohnen die im vierten Stock, da wird man halt auch mal müde [...]. Und weil es eben so ein Wettbewerb ist, sind dann halt auch besonders ehrgeizige Personen in Positionen der Teamleitung.

Fahrerin B 2018

Durch eine solche algorithmisch induzierte Quantifizierung der Arbeitsleistung und lückenlose Ablaufkontrolle wird eine bislang ungekannte Form der Arbeitstransparenz hergestellt (Shapiro 2018; Griesbach u.a. 2019). Das automatisierte Tracking dokumentiert eine detaillierte individuelle ,Leistungsübersicht', die jede auf der Plattform registrierte Aktivität - auch jeden Unfall und Konflikt - als Referenz für künftige Tätigkeiten speichert. Die vermeintliche Objektivität der Daten lässt dabei außen vor, wie die jeweiligen Kennziffern genau zustande kommen und welche Aussagekraft ihnen zukommt. Das algorithmische Management bleibt für die Arbeiter ${ }^{\star}$ innen eine black box, durch die sich die Informationsasymmetrie zwischen dem arbeitgebenden Unternehmen und den im Prinzip jederzeit auswechselbaren Arbeitskräften beständig erweitert (Kellogg u.a. 2020). Darüber hinaus verschärft der permanent mögliche Leistungsvergleich anhand quantifizierter Kennziffern den Konkurrenzdruck unter den Fahrer`innen ebenso wie die kleinteilige automatisierte Arbeitskontrolle, die die (eigentlich beworbene) Autonomie der Arbeitnehmenden unterminiert (Jarrahi u.a. 2020).

\subsection{Widerstand und Selbstorganisation der Fahrer*innen}

Das algorithmische Arbeitsmanagement war aus Sicht der interviewten Fahrer^innen allerdings lange Zeit nicht das vorrangige Problem. Vielmehr wurde bemängelt, dass viele Arbeitsmittel selbst eingebracht werden müssten, die Bezahlung zu niedrig angelegt sei und selbstverständliche arbeitgeberseitige Leistungen nur unvollständig erbracht würden: „Also der Arbeitgeber will nur bezahlen, was seiner Meinung nach ,wirkliche' Arbeit ist, die man hier leistet. Und das ist immer nur die Auslieferung“ (Fahrer`in D 2018; auch: Fahrer^in C 2018). 
Gegen diese Arbeitsbedingungen formierte sich bereits ab 2016 internationaler Widerstand (Tassinari/Maccarrone 2020). In Deutschland arbeiteten ab 2017 die Freie Arbeiterinnen- und Arbeiter-Union (FAU), die Gewerkschaft Nahrung-Genuss-Gaststätten (NGG) und ver.di mit Kurierfahrenden verschiedener Unternehmen zusammen, um einen Forderungskatalog zu erarbeiten und Demonstrationen zu organisieren (Degner/Kocher 2018). Zum einen wurde eine Bereitstellung der Arbeitsmittel und die Einführung einer Betriebshaftpflichtversicherung eingefordert. Zum anderen sollten Reparaturen übernommen und die Transparenz bei der Schichtplanung erhöht werden. Das übergreifende Ziel besteht nach wie vor in der Etablierung eines Tarifvertrags.

Die Wirkungen der Proteste hielten sich zunächst in Grenzen: Während Deliveroo jegliche Verhandlungen ablehnte, ließ sich Foodora 2017 symbolisch auf eine Gesprächsrunde ein. In den Verhandlungen zwischen der FAU Berlin und der Foodora-Geschäftsführung wurde die Ausarbeitung eines Modells zur gestaffelten Entgelterhöhung und eine verbesserte Transparenz in der Schichtplanung vereinbart. Es blieb jedoch bei mündlichen Zugeständnissen, die danach in der Presse durch den damaligen Foodora-Pressesprecher Vincent Pfeiffer als „überzogene und unhaltbare Forderungen“ zurückgewiesen wurden (zit. n. Kramer 2017). Ab Anfang 2018 kompensierte Foodora den Verschleiß der Arbeitsmittel durch eine Kilometerpauschale von 25 Cent. Diese Sachmittelzuweisung musste indes bei einer vorgegebenen Werkstätte eingelöst werden (FAU 2018). Arbeitnehmer*innenvertretungen gegenüber legte Foodora Deutschland vordergründig zwar eine indifferente Haltung an den Tag. Dennoch gab es bis Ende 2018 nur an zwei von 34 Standorten einen Betriebsrat.

Bei Lieferando.de operierten Mitte 2020 an sechs Standorten gewählte Betriebsräte; inzwischen wurden in gut einem Dutzend deutschen Städten Arbeitnehmer^innenvertretungen installiert (Stand: Oktober 2021). Allerdings zeigte sich das Management von Lieferando.de im Untersuchungszeitraum abweisend gegenüber Mitbestimmungsstrukturen und torpedierte Betriebsratswahlen, wann immer dies ohne öffentliches Aufsehen möglich war:

So, ich bin jetzt seit vier Jahren dabei und all die Erfolge, die wir bei Foodora hatten, die wir auch verschriftlichen wollten, weil es immer so viele Veränderungen gibt, aber wir haben es nicht geschafft, die in ordentliche Vereinbarungen zu pressen. Und jetzt fangen wir bei Lieferando wieder von vorne an, aber mit einem Management, das uns sehr stark bekämpft.

Fahrer*in F 2020

Wie schon bei Foodora wurden Betriebsräte durch Lieferando.de in ihrer Arbeit regelmäßig behindert. Sie wurden ignoriert, nicht rechtzeitig über technische oder logistische Umstellungen informiert oder - so berichteten Interviewpart- 
ner`innen - zum Teil mit Einschüchterungsversuchen belangt. Insofern verwundert es nicht, dass die Betriebsratsarbeit zunehmend durch gerichtliche Beschlüsse eingeklagt wird und so auf dem juristischen Weg einige Erfolge, wie etwa jüngst die Entfristung aller Arbeitsverträge, erreicht wurden (SZ 2021).

\section{Plattformnutzung in kooperativen Arbeitszusammenhängen}

Als Gegenpol zum algorithmischen Management in der Gig Economy lassen sich neben plattformbasierten Projektzusammenhängen wie Open-Source-Communities (Schrape 2019) mehr oder minder gemeinwohlorientierte bzw. sozialreformerische Formen des plattformvermittelten Ko-Konsums unter dem Begriff der ,solidarischen Ökonomie، (Voß 2010; Kerber-Clasen 2012) subsumieren. Zum ersten ist das die basisdemokratisch organisierte Initiative Foodsharing.de, die 2012 gegründet wurde und Lebensmittelverschwendung bekämpfen will. Die Plattform wirkt hier als kollaborationsermöglichende Infrastruktur, während Vertrauen und soziale Beziehungen über persönliche Begegnungen hergestellt werden. Zum zweiten sind das soziale Kollektive, die tendenziell im AllmendeKontinuum zu verorten sind, darunter der 2011 gegründete Verein solidarische Landwirtschaft e.V., der sich als Vernetzungsplattform für Betriebe und Haushalte versteht. Zum dritten sind das Projekte aus dem Bereich Social Entrepreneurship, die sich wie die 2017 gegründete SIRPLUS GmbH in der Selbstbeschreibung im Allmende-Kosmos verorten, aber realiter wie Start-up-Firmen agieren (Schreyer 2019).

In der Betrachtung dieser Spielarten des plattformvermittelten Ko-Konsums tritt hervor, dass es über die Implementation projektspezifischer Plattformarchitekturen hinaus auf die soziale Aushandlung kollektiv akzeptierter Organisationsprinzipien ankommt, die in Konfliktfällen Orientierung bieten. Als Gegengewicht zu der anonymen Koordination durch algorithmische Plattformstrukturen bleiben persönliche Abstimmungsprozesse als gemeinschaftsstiftendes Element wichtig, welche vor Ort oder auf den Plattformen selbst stattfinden können. In der kommerziellen Gig Economy wie auch auf den Sharing-Plattformen des Social Entrepreneurship sind die Optionen dazu jedoch kaum angelegt. Zudem zeigt sich, dass im Zeitverlauf ökonomische Rahmenbedingungen an Relevanz gewinnen: Während zu Beginn intrinsische Motivationen im Vordergrund stehen, wird mit zunehmender Projektdauer auf individueller Ebene eine (mo- 
netäre) Gratifikation ebenso notwendig, wie auf kollektiver Ebene die selbsttragende Wirtschaftlichkeit immer relevanter erscheint (Schreyer/Schrape 2021).

Eine mögliche Organisationsform für gemeinwohlorientierte Projektzusammenhänge, die diese drei Aspekte (Absicherung, kollektive Abstimmung, Wirtschaftlichkeit) adressiert, findet sich in dem Konzept der Genossenschaften, die als die „älteste Form der Sharing Economy“ beschrieben werden (Gerling 2018). Genossenschaften sind eng an ihre Werte gebunden und entfalten so eine identitätsstiftende Wirkung. Dabei ist es wichtig, zwischen juristischer Rechts- und sozialer Organisationsform zu unterscheiden. Genossenschaftliche Organisationsformen lassen sich entlang dreier Prinzipien identifizieren (Bolsinger 2006): Der Betrieb befindet sich im Eigentum der Mitglieder, die aktiv in alle Entscheidungsprozesse eingebunden sind (Identitätsprinzip). Alle Mitglieder sind gleichberechtigt und übernehmen abwechselnd anfallende Aufgaben (Demokratie- und Rotationsprinzip). Die Gewinnerzielungsabsicht leitet sich aus dem Anspruch ab, dass jedes Mitglied seinen Lebensunterhalt bestreiten kann (Subsistenzprinzip).

Als ein Beispiel für einen plattformkoordinierten Arbeitszusammenhang, der nach den skizzierten genossenschaftlichen Prinzipien strukturiert ist (aber rechtlich nicht als Genossenschaft auftritt), nehmen wir im Folgenden das Lastenradkurierkollektiv Crow Cycle Courier Collective (Crow) explorativ in den Blick. Die Betrachtungen basieren angesichts weniger sozialwissenschaftlicher Studien zu dem Projekt selbst wie auch zu sozialreformerischen kollektiven Arbeitsformen insgesamt (Kerber-Clasen 2012, 283) zum einen auf der qualitativen Auswertung von Zeitungsartikeln, Webinhalten sowie Projektdokumenten über das Kollektiv (2017-2021). Zum anderen wurden nach einem zunächst schwierigen Feldzugang zwei ausführliche Face-to-Face-Interviews mit zwei Mitgliedern von Crow sowie vier Telefoninterviews und zwei informelle Hintergrundgespräche via E-Mail bzw. im Kontext einer Online-Tagung durchgeführt. Die Gespräche wurden in englischer und deutscher Sprache geführt und dauerten 10 bis 270 Minuten. Die geplante teilnehmende Beobachtung und weitere Interviews vor Ort konnten aufgrund der COVID-19-Pandemie nicht mehr stattfinden. Darüber hinaus wurde ein Podcast-Interview mit vier weiteren Mitgliedern des Kollektivs aus dem Jahr 2019 in die Analyse aufgenommen. Das Durchschnittsalter der Interviewten lag bei 28,9 Jahren. Die Auswertung des Datenmaterials wurde in derselben Weise vorgenommen wie in Kapitel 3 dargestellt. 


\subsection{Grundstrukturen des Crow Cycle Courier Collective}

Das Lastenradkurierkollektiv wurde 2017 durch sechs Menschen in Berlin gegründet, die sich durch ihre Arbeit für Deliveroo kannten. Das Initialmoment wird zum einen in der Forderung nach besseren Arbeitsbedingungen und in einer Überwindung der Atomisierung der Fahrerinnen verortet (Wewer 2016). Zum anderen ging es den Initiator*innen darum, die aus ihrer Sicht gegebenen Vorteile des freiberuflichen Daseins zu erhalten. Die Kurierkooperative ist selbstverwaltet und eigenverantwortlich organisiert. Sie versteht sich als dezentrales Netzwerk und Projektunternehmen, das für andere lokale Unternehmen tätig wird, die nachhaltige Lieferketten aufbauen wollen (Crow 2020a, 2020b).

Das Crow Cycle Courier Collective ist als Gesellschaft bürgerlichen Rechts (GbR) eingetragen. Die zwei Gesellschafter`innen haben abgesehen von Verwaltungsgebühren weder Fremd- noch Eigenkapital eingebracht. Diese Unterkapitalisierung in den Anfangsjahren stellt generell eine Herausforderung für selbstverwaltete Betriebe dar (Zölch/Peters 1988). Die Ideenfindung und Organisation der Gründung basierte auf freiwilliger Arbeit und nahm fast ein Jahr Vorlauf in Anspruch; aufgrund des fehlenden Kapitals sei es nicht notwendig gewesen, vor der tatsächlichen Gründung „einen offiziellen Rechtskörper zu formen“ (Bike Messenger 3, 2019) ${ }^{2}$. Auch diese Dynamik scheint typisch für selbstverwaltete Betriebe zu sein: In der Anfangsphase ist die Bereitschaft zur Leistung unbezahlter Arbeit hoch. Diese kann aber nicht mit der gleichen Konsequenz wie bezahlte Arbeit ausgeübt werden, weshalb sich die Ausarbeitung der Arbeitsstrukturen oftmals verzögert (Berger 1986).

Obgleich es in Berlin bereits ein Kurierkollektiv (Fahrwerk UG, gegründet 2009) gab, wollten die Gründer^innen von Crow etwas Neues kreieren, um ihre „eigenen Ideen und Vorstellungen“ (Bike Messenger 3, 2019) zu verwirklichen. Ein weiterer Grund für die Neugründung bestand darin, dass nach der Beobachtung der Initiator*innen in längerfristig bestehenden Kollektiven oft informelle Hierarchien entstehen. Von anderen Kurierdienstleistern setzt sich Crow bereits durch die Eigenbezeichnung der Fahrer^innen ab, die als ,Bike Messenger firmieren und sämtliche Aufgaben im Kollektiv übernehmen. Crow orientiert sich insofern an genossenschaftlichen Organisationsformen. Das Kollektiv praktiziert eine basisdemokratische Selbstverwaltung, bei der das Identitätsprinzip (alle Mitglieder beteiligen sich an der Willensbildung), das Rotationsprinzip (je-

2 „Bike Messenger“ ist die Selbstbezeichnung der Fahrer`innen von Crow. Die Klammer steht für die jeweiligen Interviewpartner`innen. 
de $^{\star}$ r kann jede Aufgabe übernehmen) und das Subsistenzprinzip (jedes Mitglied kann seinen Lebensunterhalt bestreiten) im Vordergrund stehen.

\subsection{Prinzipien in der kollektiven Arbeitsorganisation}

2020 waren 15 Menschen im Alter von 26 bis 40 Jahren in das kooperative Unternehmen involviert. Während prinzipiell alle Mitglieder an der kollektiven Selbstverwaltung partizipieren, waren in unserem Beobachtungszeitraum (Mitte 2019 bis Anfang 2021) nur acht Arbeiter*innen in Vollzeit für das Kollektiv aktiv. Dies liegt zum einen darin begründet, dass viele Mitglieder daneben anderen beruflichen Tätigkeiten nachgehen. Die jeweils inaktiven Mitglieder nehmen jedoch regelmäßig an den Plenarsitzungen teil und verfügen über ein gleichrangiges Stimmrecht gemäß dem Identitäts- und Demokratieprinzip. Zum anderen konnte das Kollektiv Anfang 2020 auch nur acht Personen in Vollzeit bezahlen. Davon waren sechs Personen jeden Tag involviert; daneben gab es eine Person, die ausschließlich in der Buchhaltung tätig war. Solche spezialisierten Positionen als Ausnahme von dem verfolgten Rotationsprinzip scheinen typisch für kollektiv geführte Unternehmen zu sein (Voigt-Weber 1993).

Crow ist eine Kooperative von Soloselbstständigen und verfügt über keine eigenständigen Büroräume. Im Crow Cycle Courier Collective gibt es keine formalen Arbeitsverträge. Vielmehr gilt ein ungeschriebener sozialer Kontrakt, auf den man sich bei Einstieg in das Projekt implizit einlässt. Da allgemein bekannt sei, dass das Kollektiv auf aktiver Partizipation und Freiwilligkeit beruhe, werde - so unsere Gesprächspartner*innen - das Engagement über die bezahlte Erwerbsarbeit hinaus als selbstverständlich angesehen (Bike Messenger 5, 2020; Bike Messenger 6, 2020). Im Sinne der Wirtschaftlichkeit mag dies notwendig sein, dennoch plädieren einige Mitglieder dafür, dass Crow die Arbeitnehmenden ab einem gewissen Punkt regulär mit den üblichen Arbeitnehmerschutzrechten einstellen sollte. Obwohl sich die Kooperative politisch gegen die Ausbeutung von Arbeiter*innen positioniert, zahlt auch Crow realiter bislang im Schnitt lediglich einen Stundenlohn in Mindestlohnhöhe. Darüber hinaus müssen auch die Kosten für Reparaturen am eigenen Arbeitsmaterial größtenteils selbst übernommen werden. Da allerdings das Kollektiv gemäß dem Subsistenzprinzip selbst entscheidet, wer auf der Grundlage des erwirtschafteten Gewinns welche Entlohnung für welche Arbeit erhält, wird es aus Sicht der Interviewten seinen Ansprüchen insgesamt durchaus gerecht:

Wir nehmen alles, was am Ende des Monats insgesamt für das Monat reingekommen ist, und teilen das entsprechend durch die Stunden, die die Menschen auf der Straße oder in 
der Dispo oder sonstigen Arbeit gemacht haben, und dann, ja, wird das, so gut wie wir können mit dem, was wir haben, aufgeteilt.

Bike Messenger 1, 2019

Damit Crow seinen Mitgliedern dauerhaft eine lebenswerte Vergütung zahlen kann, muss das Unternehmen weiter wachsen. Derzeit werden im Sinne der Ertragsarbeit 20 Prozent des Betrags, die ein Auftrag erwirtschaftet, in die Kollektivkasse eingezahlt. Dieses Geld wird für laufende Kosten und Steuerzahlungen verwendet. Während andere Kurierdienste mit diesem Geld auch die Büroverwaltung finanzieren, ist dies bei Crow nicht der Fall, da fast alle Bike Messenger auch Verwaltungsaufgaben übernehmen. Die Aufgaben werden nach dem Grundsatz einer „risikogerechten Bezahlung“ (Crow 2020a) unterschiedlich vergütet:

\footnotetext{
Wir zahlen auch nicht jede Arbeit gleich aus. Ja wir zahlen ungleiche Arbeit nicht gleich aus. [...] Und ja, ich sitze zu Hause und irgendjemand anders hängt auf der Straße, zwischen zwei SUVs und irgendeinem dämlichen Laster von links und rechts und äh, ohne Witz, Fahrradkurierfahren ist nicht ungefährlich. So dann seh' ich als Disponent nicht ein, dass ich genauso viel bekomme sollte wie die Person, die da draußen ihr Material kaputt macht und ihr Leben riskiert auf gewisse Art und Weise.
}

Bike Messenger 3, 2019

Dabei ist es den interviewten Mitgliedern des Kollektivs wichtig festzuhalten, dass gemäß dem Rotationsprinzip sämtliche Mitglieder jederzeit alle Aufgaben übernehmen könn(t)en. Es gebe im Kollektiv, so die Selbsteinschätzung der Interviewten, keine formale Hierarchie und keine fixierte Arbeitsteilung. Allerdings lässt sich beobachten, dass sich auch im Falle von Crow mit der Zeit gewisse Routinen und Kompetenzzuschreibungen sowie Wortführerschaften und Fraktionierungen in den Plenarsitzungen eingespielt haben.

\subsection{Kommunikation als zentrales Koordinationsprinzip}

Die Basis des Kollektivs ist intensive Kommunikation. Die politische Grundidee besteht in der Ermöglichung selbstbestimmter Arbeit durch eine Verteilung der Gestaltungsmacht auf alle Mitglieder. Kollektiv akzeptierte Regelsetzungen werden idealiter über Konsens, realiter via Mehrheitsentscheidungen hergestellt. Im Sinne des Identitäts- und Demokratieprinzips ist das kommunikative Involvement aller Mitglieder der größte Kontrast zu den kommerziellen Arbeitszusammenhängen der Gig Economy, in denen die Arbeitsorganisation zentral definiert wird und ohne Einflussmöglichkeiten für die Beschäftigten abläuft. Demgegenüber will Crow durch seine offengehaltene Kommunikationsstruktur 
einerseits die Selbstbefähigung seiner Mitglieder befördern, die sich jederzeit aktiv in die Gestaltung der Spielregeln der alltäglichen Arbeit einbringen können. Andererseits ermögliche die aktive Teilhabe aller Mitglieder laut unseren Gesprächspartner`innen auch die bestmögliche Arbeitsorganisation.

Während die alltägliche Kommunikation primär über Onlinedienste und -plattformen stattfindet, finden strategische Plenarmeetings im zweiwöchentlichen Takt statt. Alle grundsätzlichen Entscheidungen werden kollektiv im Plenum verhandelt: „Es ist ein hoher Kommunikationsaufwand, aber man muss das Plenum respektieren, als die Instanz, dann funktioniert das auch“ (Bike Messenger 1, 2019). Insgesamt attestieren alle Befragten eine hohe Kommunikationsdichte im Kollektiv. Diese sei essenziell, da nur so ein angemessener Überblick über die individuellen und kollektiven Belange behalten werden könne. Neben diesen internen Abstimmungsprozessen ist Crow in einem ständigen Austausch mit anderen Kooperativen. Crow ist seit 2018 aktives Mitglied im gemeinwohlorientierten Fahrradkurier-Netzwerk Coop Cycle, das als ,Kooperative der Kooperativen' 2016 in Frankreich und Belgien gegründet wurde und technische wie auch rechtliche Rahmenbedingungen für seine Mitglieder erarbeitet. Crow partizipiert zweimal jährlich an den Treffen, die Coop Cycle in Brüssel ausrichtet. Das Ziel dieser Treffen besteht neben Erfahrungsaustausch in der kollektiven Weiterentwicklung der Coop-Cycle-Plattform für Kurierdienste sowie in der übergreifenden Vernetzung des Versands.

Crow verwendet zur Arbeitskoordination bisher nur kostenlose Softwareprodukte, um die laufenden Kosten gering zu halten. Die zentrale Software ist die genannte Plattformapplikation von Coop Cycle (Bike Messenger 6, 2020: „without coop nothing works“). An zweiter Stelle steht der Messaging-Dienst Slack, der für die situative Abstimmung eingesetzt wird. Ebenfalls wichtig ist die Walkie-Talkie-App Zello, die Push-to-Talk-Kommunikation ermöglicht. Über Zello kommunizieren Dispatcher und Messenger während des Auslieferungsprozesses. Für die Vorbereitung der Plenartreffen wird u.a. Google Drive (Filehosting) eingesetzt.

Das Crow Cycle Courier Collective darf - wie alle anderen angeschlossenen Kooperativen - nicht direkt auf den Code der Software von Coop Cycle zugreifen, kann aber beständig Feedback geben und Probleme mit der Plattform schildern. Die Entwickler*innen von Coop Cycle bearbeiten die gemeldeten Probleme in der Regel zeitnah. Darüber hinaus gibt es ein Online-Portal für die generelle Weiterentwicklung der Software. Dort können alle Nutzer`innen Veränderungsanfragen stellen, die dann durch die Community evaluiert werden. „Wenn du Coop Cycle nutzt, dann bist du Coop Cycle. Du hast ein Mitbestimmungsrecht“ (Bike Messenger 4, 2019). Zudem können sich die an Coop Cycle ange- 
schlossenen Projekte auch entscheiden, bestimmte Funktionen der Plattform nicht einzusetzen. Beispielsweise ermöglicht die Applikation von Coop Cycle in ihrer Grundanlage auch Livetracking in Echtzeit (Coop Cycle 2020). Diese Funktion nutzt Crow jedoch bewusst nicht, da das Kollektiv die Ansicht vertritt, dass sich durch Livetracking die Stressbelastung für die Fahrer*innen erhöhe, zumal Kund`innen nie wissen könnten, wann diese etwa eine Pause bräuchten oder einen Platten hätten (Fiedler 2019).

Obgleich die alltägliche Arbeit ohne die Coop-Cycle-Applikation nicht denkbar wäre, steht der Algorithmus in der alltäglichen Arbeit von Crow also nicht als alleiniger zentraler Koordinationsmechanismus im Vordergrund. Vielmehr gründet die Funktionsfähigkeit des kooperativen Unternehmens auf dem $\mathrm{Zu}$ sammenspiel aller verwendeten kommunikationstechnischen Tools sowie intensiver kommunikativer Abstimmung. Aufgrund des geringen Maßes an technikvermittelter Standardisierung gibt es den Interviewten zufolge auch keinen typischen Tagesablauf: Für gewöhnlich müssen die Mitglieder sehr flexibel sein, da die Arbeitsabläufe oft nicht eindeutig definiert sind. Daneben treten regelmäßig Probleme auf, die eine situative Abstimmung notwendig machen, wie etwa Transportschäden oder ausgefallene Server.

\section{Plattformnutzung in kommerziellen und kooperativen Kontexten}

Die hier diskutierten Fahrradkurierdienste operieren auf einem mehrseitigen Markt (Lieferando.de) bzw. entlang einer klassischen Angebot-Nachfrage-Struktur (Crow Cycle Courier Collective). Gemeinsam ist beiden Fahrradkurierdiensten, dass die involvierten Teilnehmerinnen (Kund ${ }^{\star}$ innen, Kurierinnen, Anbieter`innen) plattformvermittelt und triangulär miteinander interagieren (Richardson 2020). Wie sich die plattformvermittelte Koordination allerdings in concreto ausspielt, unterscheidet sich in den betrachteten kommerziellen und kooperativen Arbeitszusammenhängen in substanzieller Weise: Während die intermediären Plattformstrukturen von Lieferando.de in zentralisierter Form zwischen Restaurants und bestellenden Kund*innen vermitteln wie auch die automatisierte Allokation der Auslieferungsleistungen und deren Kontrolle übernehmen, werden die Aufträge im Arbeitskontext des Crow-Kollektivs dezentral in die Coop-Cycle-Plattform eingebucht, welche auch nur einen möglichen Koordinations- und Kommunikationskanal in der alltäglichen Abstimmung darstellt. 
Im Falle von Lieferando.de ist die zentrale Plattform sowohl für die Restaurants, die dort ihr Angebot präsentieren, als auch für die Kund`innen, die dort das gewünschte Essensangebot nachfragen, der erste Anlaufpunkt. Für die Nutzung der Plattform zahlt das Restaurant eine Provision von 12 Prozent. Wenn die Fahrer*innenflotte von Lieferando.de die Auslieferung übernimmt, zahlt das Restaurant eine Umsatzprovision von 30 Prozent an das Unternehmen. Nach dem erfolgreichen ,Matching ' wird der Auftrag algorithmisch automatisiert koordiniert, das Bestellvolumen berechnet und auf die Fahrenden einer Schicht umgelegt. Gleichzeitig wird die Strecke ermittelt und die Fahrer*innen bekommen den Auftrag und die Adresse des Restaurants zugesendet. Wenn dort das Essen in Empfang genommen wurde, wird dies in der App bestätigt und die Lieferadresse freigegeben. Nach der Auslieferung an die Kund ${ }^{\star}$ in bekommt die Fahrer^in entweder einen neuen Auftrag oder fährt zu einem Wartepunkt (Abbildung 1).

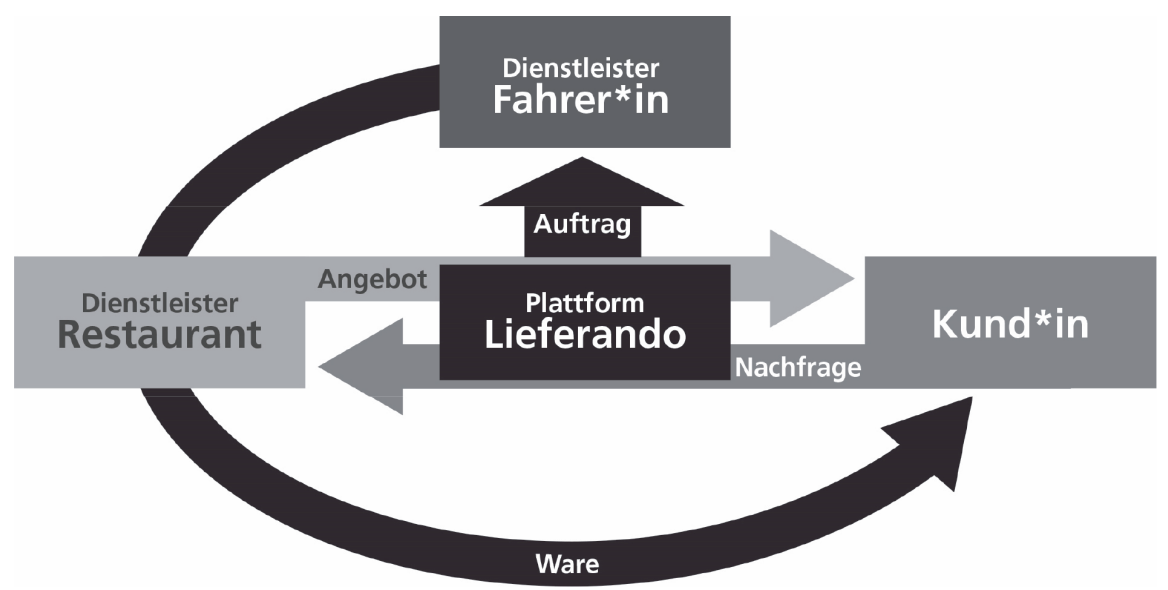

Abb. 1: Grundsätzliche Funktionsweise von Lieferando.de (eigene Darstellung)

Eine solche vollautomatisierte modularisierte Arbeitskoordination schränkt nicht nur den situativen Handlungsspielraum für Erwerbstätige erheblich ein auch wenn es um die Klärung von konkreten Ablaufschwierigkeiten geht -, sondern beschneidet die Autonomie der Arbeitnehmenden überdies in einem deutlich weiteren Sinne (Vallas/Schor 2020). Das gilt insbesondere für Arbeitskraftanbietende ohne weitergehende Qualifikationen: Solche volatil Beschäftigten werden in der Tendenz zu informationstechnisch ausgeleuchteten Ausführungsvariablen, die durch umfassende Leistungskontrollen und Plattformregularien 
eingehegt werden. Allerdings sind die Arbeitskräfte nicht nur passive Rezipient ${ }^{\star}$ innen dieser Kontrollstrategien, sondern entwickelten vielfach eigen- und widerständige Reaktionen darauf (Bucher u.a. 2021; Jarrahi u.a. 2020). Eine kollektive Mobilisierung wird zwar auch im Falle von Lieferando.de durch die anonyme Plattformkoordination grundsätzlich erschwert. Trotzdem aber wurden in den letzten Jahren Prozesse der Selbstorganisation unter den Fahrer*innen auf regionaler, nationaler und transnationaler Ebene angestoßen, die in öffentlichkeitswirksame Proteste mündeten.

Im Gegensatz zu der geschilderten Zentralstellung der Plattform in der Arbeitskoordination von Lieferando.de ist die informationstechnische Plattform Coop Cycle im Falle des kooperativen Unternehmens Crow in einen breiteren Koordinationszusammenhang eingebunden, der auf zahlreichen weiteren Kommunikationsdiensten fußt. Über die Plattform Coop Cycle, die in ihrer Funktionsweise kommerziellen Varianten durchaus ähnlich ist, werden zwar ebenfalls sämtliche Auslieferungsprozesse koordiniert. Sie bietet allerdings deutlich höhere situative Spielräume und ist weniger modular aufgebaut: Über Coop Cycle werden alle Auftragsabschlüsse, abzuarbeitende Aufträge sowie weiterführende Informationen distribuiert und koordiniert. Gleichzeitig werden die gerade bearbeiteten Aufträge durch einen Dispatcher persönlich über die WalkieTalkie-App an die Fahrer^in weitergegeben. Die Kund ${ }^{\star}$ innen kommen nur insofern mit der Plattform in Berührung, als sie die erfolgreiche Zustellung in der Smartphone-Applikation mit ihrer Unterschrift bestätigen. Sobald der Dispatcher Zugriff auf die Unterschrift hat, markiert er den Auftrag auf der Plattform als abgeschlossen. Im Gegensatz zu einem vollautomatisierten algorithmischen Management kann so unmittelbarer und kontextsensibel auf etwaige Umwelterfordernisse reagiert werden (Abbildung 2).

Anders als in der kommerziellen Gig Economy stellt die persönliche Kommunikation nicht nur in diesen Belangen die primäre Funktionsgrundlage des kooperativen Unternehmens dar. Coop Cycle als informationstechnische Plattform fungiert zwar als ein wesentlicher, aber eben nicht alleiniger Koordinationsmechanismus in den Auslieferungsprozessen. Daneben haben die Fahrer^innen die Möglichkeit, über andere Kommunikationskanäle untereinander und mit der Versandzentrale zu interagieren. Durch diese vielschichtige Infrastruktur, die sich nicht auf einen alles koordinierenden Algorithmus reduzieren lässt, ermöglicht Crow einen kontinuierlichen Austausch auf allen Arbeitsebenen. Die genutzten technischen Strukturen erleichtern die interne Koordination; sie prägen das Kollektiv aber nicht in seiner grundsätzlichen Struktur und bleiben ein Objekt sozialer Aushandlung. Darüber hinaus versucht das kooperative Unternehmen, die kapitalistische Logik zumindest teilweise außer Kraft zu 
setzen, indem es explizit auch die nicht-ökonomischen Bedürfnisse seiner Mitglieder adressiert - darunter Arbeitszufriedenheit und Mitbestimmung. Diese soziale Rationalität, die (zumindest rhetorisch) über die ökonomische Rationalität gestellt wird, bietet einerseits den Nährboden für eine hohe Identifikation mit dem Kollektiv. Andererseits geht damit jedoch auch ein hoher moralischer Druck einher, sich über die reguläre Arbeitszeit hinaus für das Kollektiv einzusetzen.

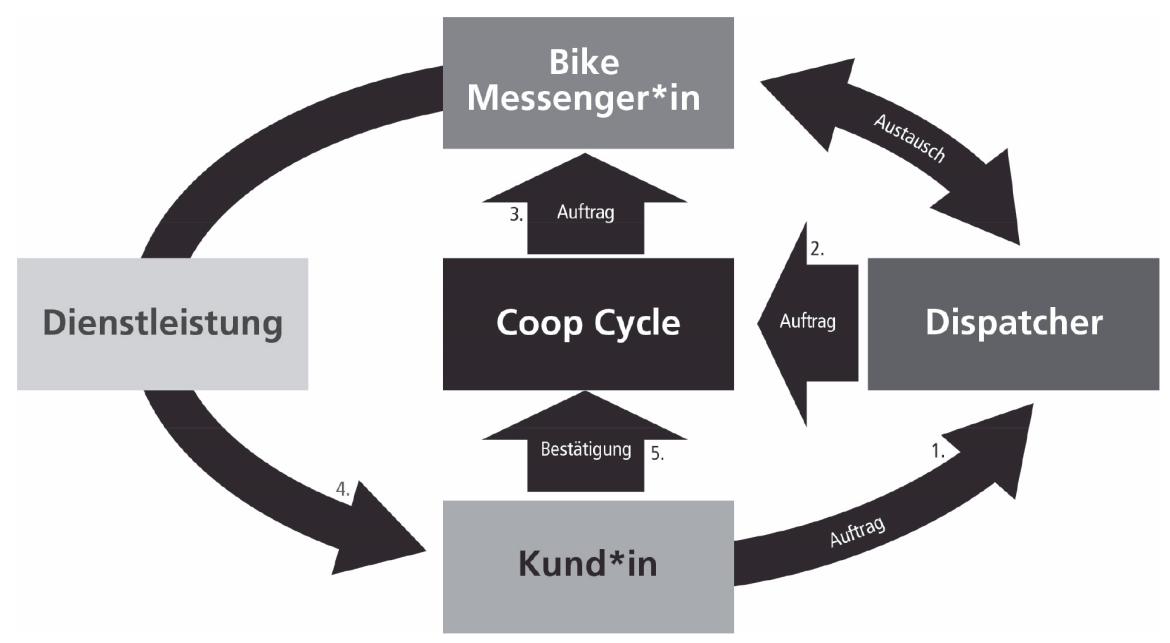

Abb. 2: Grundsätzliche Funktionsweise von Crow (via Coop Cycle) (eigene Darstellung)

Wie sich die Plattformisierung von Arbeitszusammenhängen ausspielt und welche der ermöglichenden, strukturierenden und kontrollierenden Eigenheiten informationstechnischer Plattformen fallweise hervortreten, hängt insoweit - das führt der hier vollzogene Vergleich von Lieferando.de und dem Crow Cycle Courier Collective exemplarisch vor Augen - vor allem anderen von den konkreten Implementationsweisen und den in den jeweiligen Arbeitszusammenhängen gegebenen sozioökonomischen Grundkonstellationen ab. Eine offen gehaltene informationstechnische Infrastruktur, die von ihren Nutzer^innen angepasst werden kann, mehrere komplementäre Kommunikationskanäle anbietet und in horizontal angelegte Arbeitszusammenhänge eingebettet ist, eröffnet vielfältige Möglichkeiten zur Mitbestimmung und Mitgestaltung. Geschlossene und proprietär betriebene Koordinationsplattformen, wie sie sich in vielen kommerziellen Zusammenhängen in der Gig Economy finden lassen, folgen hingegen rein ökonomischen Orientierungen und können den Austausch unter den Arbeit- 
nehmenden effektiv behindern, deren Atomisierung befördern und in einem weitreichenden Kontrollregime resultieren.

Gleichzeitig zeigt sich allerdings nicht zuletzt am Beispiel der arbeitspolitischen Konflikte um Lieferando.de (und neuerlich um den Lebensmittel-Lieferdienst Gorillas), dass technisch eröffnete Möglichkeitsräume der Automatisierung, Standardisierung und Kontrolle mit zunehmender sozioökonomischer Lebensdauer regelmäßig Objekt arbeitspolitischer Aushandlungsprozesse und öffentlicher Debatten werden, auf die Plattformunternehmen in der Ausgestaltung der soziotechnischen Regulationsmuster ihrer Plattformen und der anliegenden Arbeitsbedingungen in der einen oder anderen Form reagieren müssen, um ihr Geschäft einträglich zu halten.

Förderhinweis: Die diesem Beitrag zugrundeliegende empirische Forschung wurde durch die Hans-Böckler-Stiftung gefördert. Die konzeptionellen Arbeiten sind im Rahmen des durch die Deutsche Forschungsgemeinschaft e.V. geförderten Schwerpunktprogramms 2267: Digitalisierung der Arbeitswelten entstanden.

\section{Literatur}

Berger, J. (1986): Selbstverwaltete Betriebe in der Marktwirtschaft. Bielefeld: AJZ

Boes, A., T. Kämpf, B. Langes, T. Lühr, S. Steglich (2014): Cloudworking und die Zukunft der Arbeit. Kassel: BTQ

Boes, A., T. Kämpf, B. Langes, T. Lühr (2018): ,Lean‘ und ,agil‘ im Büro. Bielefeld: transcript Bolsinger, H. (2006): Die Genossenschaft als Kooperationsmodell für symbiotische KMU-Netzwerke. Nürnberg: Forschungsinstitut für Genossenschaftswesen

Bucher, E. L., P. K. Schou, M. Waldkirch (2021): Pacifying the algorithm - Anticipatory compliance in the face of algorithmic management in the gig economy; in: Organization, 28, 1, 44-67

Coop Cycle (2020): About Coop Cycle. https://coopcycle.org/en/, abgerufen Oktober 2021

Crow (2020a): Home. Crow Cycle Courier Collective. https://crowberlin.de/, abgerufen Oktober 2021

Crow (2020b): Über uns. Crow Cycle Courier Collective. https://crowberlin.de/uber-uns/, abgerufen Oktober 2021

Degner, A., E. Kocher (2018): Arbeitskämpfe in der ,Gig-Economy‘?; in: Kritische Justiz, 51, 3, 247-265

De Stefano, V. (2016): The rise of the 'just-in-time workforce'. On-demand work, crowdwork and labour protection in the 'gig-economy'. Conditions of Work and Employment Series No. 71. Genf: ILO

Dolata, U., J.-F. Schrape (2018): Collectivity and Power on the Internet. Cham: Springer 
Duggan, J., U. Sherman, R. Carbery, A. McDonnell (2020): Algorithmic Management and Appwork in the Gig Economy; in: Human Resource Management Journal, 30, 1, 114-132

Eichhorst, W., A. Spermann (2015): Sharing Economy: Mehr Chancen als Risiken?; in: Wirtschaftsdienst, 96, 433-439

FAU (2018): Special Delivery from \#deliverunion. https://deliverunion.fau.org/2018/01/29/ special-delivery-from-deliverunion/, abgerufen Oktober 2021

Fiedler, L. (2019): Fahrradkuriere: Im Kollektiv gegen die Gig-Economy; in: Zeit Online vom 27. September 2019. https://www.zeit.de/arbeit/2019-09/fahrradkuriere-crow-cycle-couriercollective-nachhaltigkeit, abgerufen Oktober 2021

Foodora (2018): About us. https://www.Foodora.com/about/, abgerufen Dezember 2018

Gerling, W. (2018): Eröffnet die kollaborative Ökonomie den Genossenschaftsbanken neue Chancen?; in: Enorm. Weconomy Spezial 2018/1, 35

Glaser, B. G., A. Strauss (1998): Grounded Theory: Strategien qualitativer Forschung. Bern: Huber

Griesbach, K., A. Reich, L. Elliott-Negri (2019): Algorithmic Control in Platform Food Delivery Work; in: Socius, 5, 4, 1-15

Healy, J., A. Pekarek, A. Vromen (2020): Sceptics or Supporters? Consumers' Views of Work in the Gig Economy; in: New Technology, Work and Employment, 35, 1, 1-19

Heiland, H., U. Brinkmann (2020): Liefern am Limit; in: Industrielle Beziehungen, 27, 1, 120-140

Hensel, I., D. Schönefeld, E. Kocher, A. Schwarz, J. Koch (Hg.) (2019): Selbstständige Unselbstständigkeit. Baden-Baden: Nomos

Huws, U. (2014): Labor in the Global Digital Economy. New York: Monthly Review Press

Huws, U. (2016): Logged Labour: A New Paradigm of Work Organisation?; in: Work Organisation, Labour \& Globalisation, 10, 1, 7-26

Huws, U., N. Spencer, D. Syrdal, N. Holts (2017): Work in the European Gig Economy Research. Results from the UK, Sweden, Germany, Austria, The Netherlands, Switzerland and Italy. Brüssel: Foundation for European Progressive Studies

Ivanova, M., J. Bronowicka, E. Kocher, A. Degner (2018): The App as a Boss? Control and Autonomy in Application-Based Management; in: Arbeit | Grenze | Fluss, 1, 1-27

Jarrahi, M. H., W. Sutherland, S. B. Nelson, S. Sawyer (2020): Platformic Management, Boundary Resources for Gig Work, and Worker Autonomy; in: Computer Supported Cooperative Work, 29, 1-2, 153-189

Kellogg, K. C., M. A. Valentine, A. Christin (2020): Algorithms at Work: The New Contested Terrain of Control; in: Academy of Management Annals, 14, 1, 366-410

Kerber-Clasen, S. (2012): Produktivgenossenschaften und solidarische Ökonomie als Forschungs- und Praxisfeld; in: WSI-Mitteilungen, 65, 4, 281-288

Kirchner, S., E. Schüßler (2019): The Organization of Digital Marketplaces; in: G. Ahrne, N. Brunsson (Hg.): Organization outside Organizations. Cambridge: Cambridge University Press, 131-154

Knieps, S. (2021): Lieferando-Deutschland-Chefin: „Wir sind nicht bloß ein Wimpernschlag in einer Coronaphase“; in: WIWO vom 9. Mai 2021. https://www.wiwo.de/unternehmen/ dienstleister/lieferando-deutschland-chefin-wir-sind-nicht-bloss-ein-wimpernschlag-ineiner-coronaphase/27036696.html, abgerufen Oktober 2021

Kramer, B. (2017): Behandelt Foodora seine Mitarbeiter fair?; in: Zeit Online vom 10. November 2017. https://www.zeit.de/arbeit/2017-11/foodora-fahrer-proteste-gewerkschaftverhandlungen, abgerufen Oktober 2021 
Kuhn, K. M., T. Galloway (2019): Expanding perspectives on gig work and gig workers; in: Journal of Managerial Psychology, 34, 186-191

Lamnek, S. (2010): Qualitative Sozialforschung. 5. Auflage. Weinheim/Basel: Beltz

Lehdonvirta, V. (2018): Flexibility in the Gig Economy: Managing Time on Three Online Piecework Platforms; in: New Technology, Work and Employment, 33, 1, 13-29

Leonardi, D., A. Murgia, M. Briziarelli, E. Armano (2019): The Ambivalence of Logistical Connectivity: A Co-Research with Foodora Riders; in: Work Organisation, Labour \& Globalisation, 13, 1, 155-171

Lücking, S. (2019): Arbeiten in der Plattformökonomie. Forschungsförderung Report 5. Düsseldorf: Hans-Böckler-Stiftung

Mayntz, R. (2002): Zur Theoriefähigkeit makro-sozialer Analysen; in: R. Mayntz (Hg.): Akteure Mechanismen - Modelle. Frankfurt a.M.: Campus, 7-43

Mayntz, R. (2009): Sozialwissenschaftliches Erklären. Frankfurt a.M.: Campus

Meyer, U., S. Schaupp, D. Seibt (Hg.) (2020): Digitalization in Industry. Cham: Palgrave Macmillan

OECD (2019): An Introduction to Online Platforms and Their Role in the Digital Transformation. Paris: OECD

Pfeiffer, S. (2018): Technisierung von Arbeit; in: F. Böhle, G. G. Voß, G. Wachtler (Hg.): Handbuch Arbeitssoziologie. Bd. 1. Wiesbaden: Springer VS, 321-358

Ravenelle, A. (2019): Hustle and Gig: Struggling and Surviving in the Sharing Economy. Berkeley: University of California Press

Richardson, L. (2020): Platforms, Markets, and Contingent Calculation: The Flexible Arrangement of the Delivered Meal; in: Antipode, 52, 3, 619-636

Rifkin, J. (2014): Zero Marginal Cost Society. New York: Putnam

Rosenblat, A. (2018): Uberland: How Algorithms Are Rewriting the World of Work. Berkeley: University of California Press

Rosenblat, A., L. Stark (2016): Algorithmic Labor and Information Asymmetries: A Case Study of Uber's Drivers; in: International Journal of Communication, 10, 3758-3784

Schäfer, Holger (2020): Crowdwork. Stellungnahme im Ausschuss Arbeit und Soziales des Deutschen Bundestages. IW-Report 63. Köln: IW

Schrape, J.-F. (2019): Open-source Projects as Incubators of Innovation; in: Convergence, 25, 3, 409-427

Schrape, J.-F. (2021): Digitale Transformation. Bielefeld: Transcript/UTB

Schreyer, J. (2019): Das Phänomen Sharing Economy am Beispiel des Foodsektors. HBS Working Paper 145. Düsseldorf: HBS

Schreyer, J. (2021a): Algorithmic work coordination and workers' voice in the COVID-19 pandemic: The case of Foodora/Lieferando; in: Work Organisation, Labour \& Globalisation, $15,1,69-84$

Schreyer, J. (2021b): Algorithmic management versus organising protest and co-determination? The case of Foodora/Lieferando in Germany; in: Studi Organizzativi, 1, 105-128

Schreyer, J., J.-F. Schrape (2018): Algorithmische Arbeitskoordination in der plattformbasierten Gig Economy; in: Arbeits- und Industriesoziologische Studien, 11, 2, 262-278

Schreyer, J., J.-F. Schrape (2021): Digitale Plattformen in kommerziellen und gemeinwohlorientierten Arbeitszusammenhängen. HBS Study 460. Düsseldorf: HBS

Schröder, M. (2016): Der Markt hat nur Platz für einen oder zwei Anbieter; in: Handelsblatt vom 4. Dezember 2016. https://www.deliveryhero.com/der-markt-hat-nur-platz-fur-einenoder-zwei-anbieter/, abgerufen Oktober 2021 
Shapiro, A (2018): Between Autonomy and Control: Strategies of Arbitrage in the 'on-Demand' Economy; in: New Media \& Society, 20, 8, 2954-2971

Srnicek, N. (2017): Platform Capitalism. Cambridge: Polity

Strauss, A., J. Corbin (1996): Grundlagen qualitativer Sozialforschung. Weinheim: Verlagsunion SZ (Süddeutsche Zeitung) (2021): Lieferando bietet Kurieren unbefristete Verträge an. Sueddeutsche.de vom 13. August 2021. https://www.sueddeutsche.de/wirtschaft/ lebensmittel-lieferando-bietet-kurieren-unbefristete-vertraege-an-dpa.urn-newsml-dpacom-20090101-210813-99-824542, abgerufen Oktober 2021

Tassinari, A., V. Maccarrone (2020): Riders on the Storm. Workplace Solidarity among Gig Economy Couriers in Italy and the UK; in: Work, Employment and Society, 34, 1, 35-54

Thelen, K. (2018): Regulating Uber. The Politics of the Platform Economy in Europe and the United States; in: Perspectives on Politics, 16, 4, 938-953

Trinczek, R. (2011): Überlegungen zum Wandel von Arbeit; in: WSI-Mitteilungen, 64, 11, 606614

Vallas, S., J. Schor (2020): What Do Platforms Do? Understanding the Gig Economy; in: Annual Review of Sociology, 46, 1, 273-294

van Doorn, N. (2017): Platform Labor: On the Gendered and Racialized Exploitation of LowIncome Service Work in the 'on-Demand' Economy; in: Information, Communication \& Society, 20, 6, 898-914

van Doorn, N. (2020): At What Price? Labour Politics and Calculative Power Struggles in onDemand Food Delivery; in: Work Organisation, Labour \& Globalisation, 14, 1, 136-149 Voigt-Weber, L. (1993): Inklusive Organisationen. Wiesbaden: DUV Voß, E. (2010): Wegweiser Solidarische Ökonomie. Neu-Ulm: leibi.de Wewer, G. (2016): Privates Teilen als Geschäftsmodell? Politische, wirtschaftliche und soziale Probleme der Sharing Economy; in: Leviathan, 44, 1, 155-179

Zölch, M., M. Peters (1988): Arbeit in Alternativbetrieben. Berlin: Universitätsverlag Zuboff, S. (2018): Das Zeitalter des Überwachungskapitalismus. Frankfurt a.M.: Campus 